\title{
Clinical Outcomes of Mechanical Thrombectomy in Stroke Tandem Lesions According to Intracranial Occlusion Location
}

\author{
Elena Zapata-Arriaza, ${ }^{\mathrm{a}}$ Asier de Albóniga-Chindurza, ${ }^{\mathrm{a}}$ Joaquin Ortega-Quintanilla, ${ }^{\mathrm{a}}$ \\ Irene Escudero-Martínez, ${ }^{\mathrm{b}}$ Francisco Moniche, ${ }^{\mathrm{b}}$ Manuel Medina-Rodríguez, ${ }^{\mathrm{b}}$ Blanca Pardo-Galiana, \\ Juan Antonio Cabezas Rodríguez, ${ }^{\mathrm{b}}$ Lucía Lebrato Hernández, ${ }^{\mathrm{b}}$ Leire Ainz, ${ }^{\mathrm{b}}$ Soledad Pérez-Sánchez, ${ }^{\mathrm{c}}$ \\ Ana Domínguez-Mayoral, ${ }^{\mathrm{c}}$ Ana Barragán, ${ }^{\mathrm{c}}$ Aurelio Cayuela, ${ }^{\mathrm{d}}$ Joan Montaner, ${ }^{\mathrm{c}}$ Alejandro González García ${ }^{\mathrm{a}}$ \\ anterventional Neurorradiology Department, Stroke Research Program, Institute of Biomedicine of Seville (IBiS)/Virgen del Rocio University \\ Hospital, Seville, Spain \\ ${ }^{b}$ Neurology Department, Institute of Biomedicine of Seville (IBiS)/Virgen del Rocio University Hospital, Seville, Spain \\ 'Department of Neurology, Virgen Macarena University Hospital, Seville, Spain \\ ${ }^{d}$ Clinical Management of Public Health, Prevention and Health Promotion Unit, Southern Health Management Area of Seville, Hospital de \\ Valme, Seville, Spain
}

\section{Dear Sir:}

Tandem lesions (TLS), depict around 20\% of acute ischemic stroke (AIS) in patients with large vessel occlusion (LVO). ${ }^{1}$ TL are poorly represented in thrombectomy clinical trials, ${ }^{2}$ leading to uncertainty in the homogenization of the therapeutic strategy. ${ }^{3}$ Clinical benefit of mechanical thrombectomy (MT) in LVO alone is clear ${ }^{2}$ and untreated AIS due to tandem occlusions generally has a poor prognosis. ${ }^{4}$ There is growing evidence about efficacy and safety of MT in TL, ${ }^{5,6}$ However, the evidence of intracranial lesion impact on endovascular treatment in TL is still lacking.

We extracted the information related to the MT performed in our tertiary hospital from a local registry carried out prospectively between January 2017 and January 2019. Patients were eligible for study if they had anterior AIS within 6 hours of symptom onset or eligibility on wake-up stroke and 6 to 24 hours, confirmed by advanced brain imaging (computed tomography [CT] perfusion). ${ }^{1}$ Eligibility criteria were (1) 18 years of age and older; (2) pre-stroke modified Rankin Scale (mRS) $0-2$; (3) vascular occlusion located in the terminal internal carotid artery (TICA), M1, and M2 segments of the middle cerebral artery with the possibility of including tandem occlusions; (4) Alberta Stroke Program Early CT Score (ASPECTS) of 6 or better overall, whereas all evaluations were assisted by a ma- chine learning-based software package (e-ASPECTS, Brainomix, Brainomix Limited, Oxford, UK); and (5) fulfilling DWI or CTP Assessment With Clinical Mismatch in the Triage of Wake-Up and Late Presenting Strokes Undergoing Neurointervention With Trevo (DAWN) ${ }^{7}$ or Endovascular Therapy Following Imaging Evaluation for Ischemic Stroke 3 (DEFUSE 3$)^{8}$ eligibility criteria on wake-up stroke and 6 to 24 hour treatment window.

All patients signed informed consent forms, including statements about the use of data for research, before treatment was carried out. Intracranial occlusion was treated first (the aspiration catheter was embedded in the clot with the aim of catching the whole clot at once) unless the carotid stenosis prevented it, forcing cervical angioplasty prior to intracranial navigation of the distal aspiration catheter. Preferred endovascular technique employed for intracranial reperfusion was distal aspiration. Our team decided to manage the endovascular treatment applying groin local anesthesia as primary option, and if needed subsequent general anesthesia or conscious sedation. Intravenous (IV) fibrinolysis (recombinant tissue plasminogen activator [rtPA]) was administered to elective patients according to local protocol. In case of rtPA administration, 250 $\mathrm{mg}$ of IV aspirin was employed at the beginning of the endovascular procedure. In patients who did not receive rtPA, 500 $\mathrm{mg}$ of aspirin was administered. Double antiplatelet therapy 
with orally administrated clopidogrel or intraarterial abciximab decision during procedure, was made according to individual basis of neurologist and neuroradiologist. The angiographic collateral grade was evaluated according to the American Society of Interventional and Therapeutic Neuroradiology/Society of Interventional Radiology Collateral Flow Grading System on baseline angiography. ${ }^{9}$ Once intracranial recanalization was achieved, treatment of the internal carotid artery (ICA) lesion was left to the discretion of the operator, taking into account residual cervical occlusion/severe stenosis or thrombus apposition after waiting for 30 minutes after intracranial recanalization achievement. In case of platelet aggregation or plaque instability, stent was placed, waiting for another 20 minutes, to monitor new complications in the vascular endoprosthesis, in which case intraarterial abciximab was administered to resolve stent-associated thrombus formation (from 2 to $8 \mathrm{mg}$ according to interventionist criteria). The procedure was terminated once stability in stent patency was achieved. Demographic, radiologic, and clinical data were collected in the prospective $A$ Registry for Thrombectomy In Stroke Therapy from Andalusia (ARTISTA) registry. Successful recanalization (modified thrombolysis in cerebral ischemia [mTICl] grade $2 b-3)$, symptomatic intracranial hemorrhage ( $\mathrm{SICH}$ ) and favorable outcome at 90 days (defined by an $m R S \leq 2$ ) rates were analyzed according to intracranial occlusion: TICA, M1, and M2. The mRS was evaluated for patients with completed follow-up.
Among 784 included patients (from January 2017 to January 2019), 22.1\% ( $n=174)$ had TL. Regarding TL subgroups, 20.1\% $(n=36)$ had ICA+TICA occlusion, 50\% ( $n=87)$ ICA+M1 occlusion, and 29.3\% ( $n=51)$ ICA+M2 occlusion. Baseline characteristics of general sample, TL versus no TL and according to intracranial occlusion are shown in Supplementary Tables 1 and 2.

We found no differences in clinical outcomes at 90 days among TL versus no TL patients. According intracranial occlusion site, ICA+TICA, ICA+M1, and ICA+M2 showed non statistical differences in clinical outcomes at 90 days compared to isolated intracranial TICA, M1, and M2 occlusion (Figure 1). Regarding successful recanalization, $\mathrm{sICH}$, and death rates we did not find statistical differences among subgroups (Figure 2). After stroke subtype adjustment, we found higher rates of successful recanalization ( $\mathrm{TICl} 2 \mathrm{~b}-3$ ) in atherothrombotic $\mathrm{TL}$ patients $(P=0.025)$. However there were no differences in $\mathrm{mRS}$ 0-2 at 90 days, death and sICH rates among TL versus no $\mathrm{TL}$ according to stroke subtype (Supplementary Table 3). Intracranial hemodynamic compensation rates according to site occlusion is specified in Supplementary Table 4.

Due to technical complexity of endovascular treatment of TL, this subgroup of stroke patients has been mostly excluded from the clinical trials. ${ }^{2}$ Nevertheless, there is a growing evidence about efficacy and safety of MT in $\mathrm{TL}_{1}^{10,11}$ with ranges of $75 \%$ to $87 \%$ of successful recanalization, $44 \%$ to $73.3 \%$ of $\mathrm{mRS}$ at 90 days, $11 \%$ to $16 \%$ of mortality, and $4.4 \%$ to $8 \%$ of sICH. ${ }^{6,10,11}$

$\mathrm{mRS}$ at 90 days according intracranial occlusion

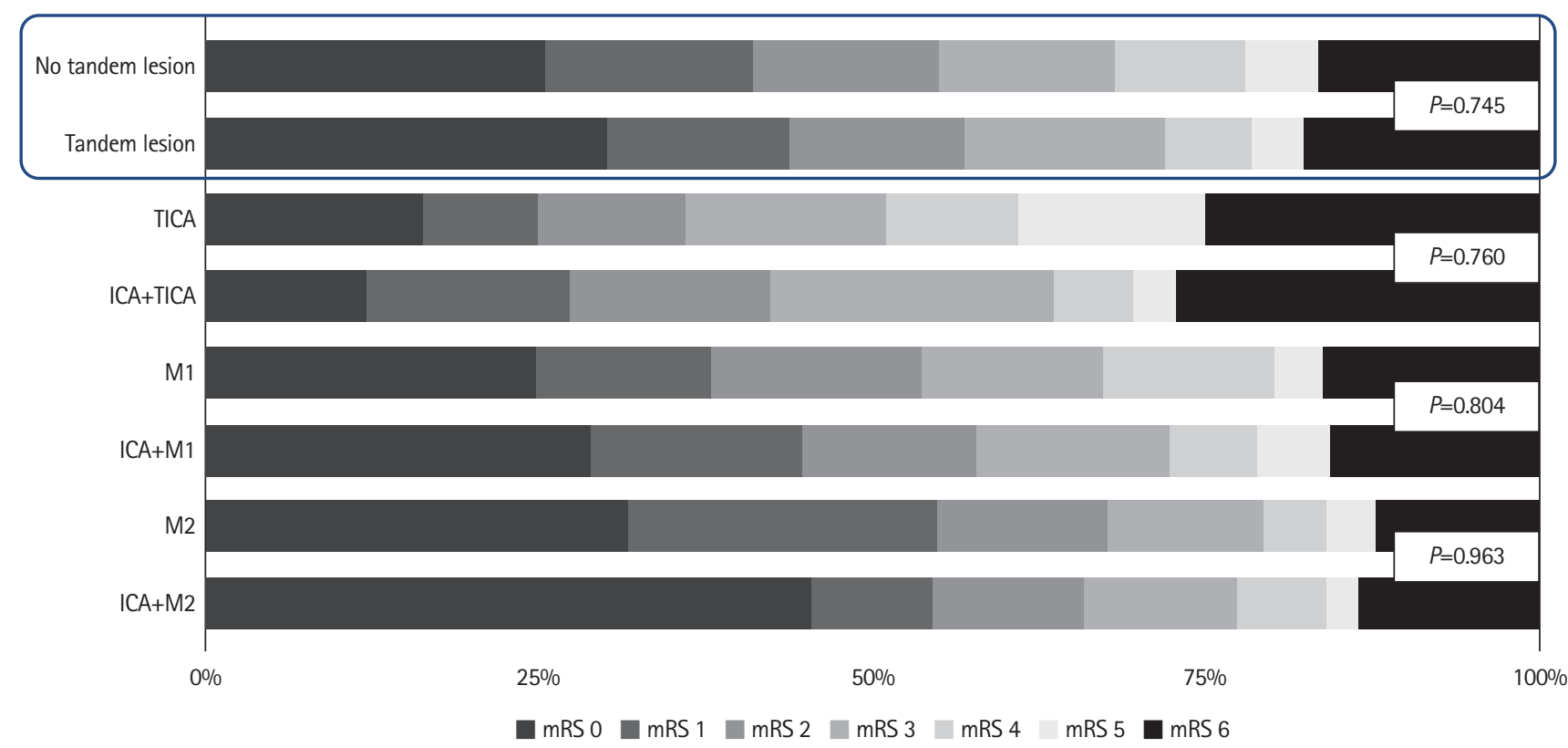

Figure 1. Modified Rankin Scale (mRS) at 90 days in tandem lesions versus no tandem lesions patients and according to intracranial occlusion. TICA, terminal internal carotid artery; ICA, internal carotid artery. 


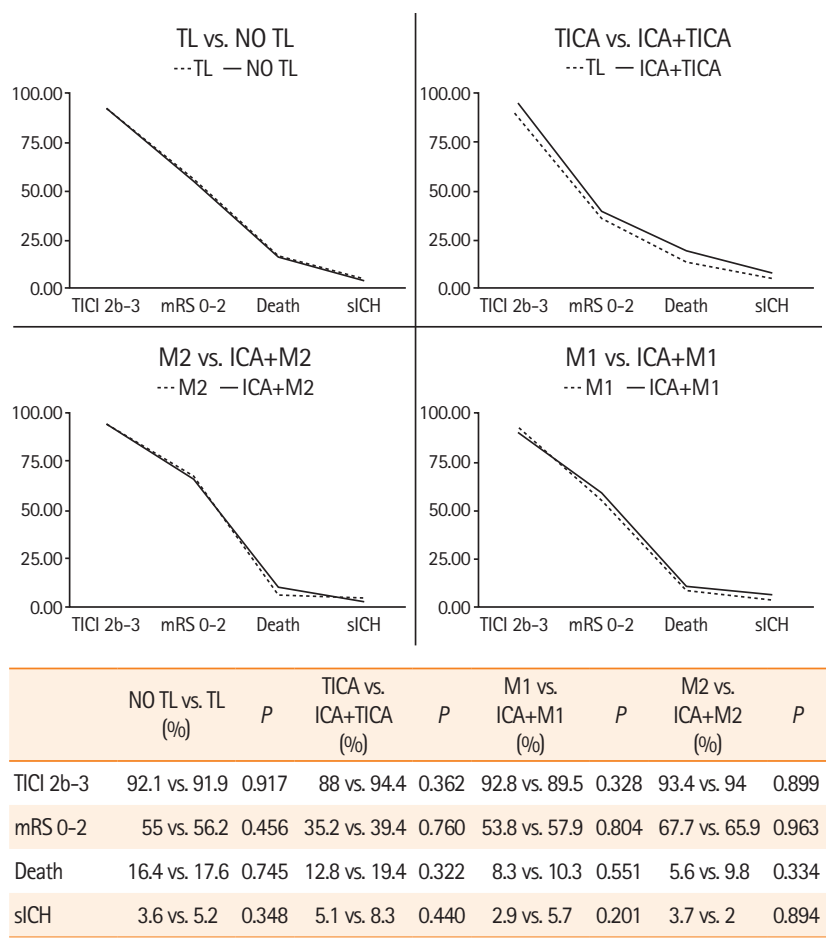

Figure 2. Safety and efficacy mechanical thrombectomy results in tandem lesions (TLS) versus no TLs patients and according intracranial occlusion. $\mathrm{TICl}$, thrombolysis in cerebral ischemia; mRS, modified Rankin Scale; $\mathrm{sICH}$, symptomatic intracranial hemorrhage; TICA, terminal internal carotid artery; ICA, internal carotid artery.

Antiplatelet therapy, endovascular approach, and stent placement in TL are still uncertain. ${ }^{12}$

There are a few recent studies related to MT results according to intracranial occlusion site, ${ }_{1}^{13,14}$ but most of them do not include $\mathrm{TL}$, which is the originality of this manuscript. Ninety days functional outcomes showed non statistical similar results when comparing $\mathrm{TL}$ and no $\mathrm{TL}$, which is consistent with results according to intracranial occlusion, downplaying importance to extracranial occlusion, whose clinical impact would be limited. In addition, it is remarkable the better results in more distal occlusions ( $\mathrm{TICA}<\mathrm{M} 1<\mathrm{M} 2)$ in both $\mathrm{TL}$ versus no TL. Furthermore, it's relevant that notwithstanding longer groin-recanalization times in all TL patients, this does not impact negatively on functional outcomes of mentioned patients. The hemodynamic compensation of the Willis circle could involve the difference between TL and no TL. In our cohort, tandem occlusion patients, have shown better intracranial compensation (retrograde ophthalmic artery, anterior communicating artery, and posterior communicating artery function), but no collateral circulation. This finding could explain that despite a longer time to obtain the successful recanalization in TLs, its functional results do not worsen and are even similar to isolated intracranial occlusions. In addition, progressive changes in intracranial hemodynamic compensation secondary to chronic significant carotid stenosis may allow the hypoperfused brain to better tolerate ischemia. According to our hypothesis, we found that cardioembolic stroke was more frequent in no TL (61.4\% vs. $11.8 \%$ ), in which acute LVO occlusion could associate worse Willis circle compensations compared to TL progressive hemodynamic changes. Main limitations of our study are the small number of patients in intracranial occlusion site subgroups and the retrospective evaluation, which introduce a selection bias for patients who met our criteria for MT.

The intracranial treatment approach in the first place proposed in our study, reduces the times of cerebral ischemia and improves the long-term functional result. Lastly, our action protocol guarantees carotid patency at the end of the procedure, since the time necessary to assess intra-stent aggregation or plaque instability is expected after administering the relevant antiplatelet agents. Ensuring cervical patency improves antegrade intracranial flow. Therefore, once intracranial recanalization has been achieved, maintaining correct carotid patency becomes essential to avoid acute reocclusions that waste the initial success.

Our results need further validation in different cohorts; however, we hypothesize that despite being more technically complex and longer procedures, patients with TL have a better tolerance to hypoperfusion, thanks to the intracranial hemodynamic compensation systems associated with a chronic carotid stenosis. This tolerance to hypoperfusion is consistent in the different intracranial occlusion sites despite presenting TLs. TL hemodynamic behavior could set a starting point to study ways of "stroke compensations" in order to reduce ischemia damage.

\section{Supplementary materials}

Supplementary materials related to this article can be found online at https://doi.org/10.5853/jos.2020.02747.

\section{References}

1. Assis Z, Menon BK, Goyal M, Demchuk AM, Shankar J, Rempel $J \mathrm{~L}$, et al. Acute ischemic stroke with tandem lesions: technical endovascular management and clinical outcomes from the ESCAPE trial. J Neurointerv Surg 2018;10:429-433.

2. Goyal M, Menon BK, van Zwam WH, Dippel DW, Mitchell PJ, Demchuk AM, et al. Endovascular thrombectomy after largevessel ischaemic stroke: a meta-analysis of individual patient data from five randomised trials. Lancet 2016;387:1723-1731.

3. Jacquin G, Poppe AY, Labrie M, Daneault N, Deschaintre $Y$, Gioia LC, et al. Lack of consensus among stroke experts on 
the optimal management of patients with acute tandem occlusion. Stroke 2019;50:1254-1256.

4. Rubiera M, Ribo M, Delgado-Mederos R, Santamarina E, Delgado $\mathrm{P}$, Montaner J, et al. Tandem internal carotid arteryl middle cerebral artery occlusion: an independent predictor of poor outcome after systemic thrombolysis. Stroke 2006;37: 2301-2305.

5. Bücke $P$, Aguilar Pérez $M$, AlMatter $M$, Hellstern $V$, Bäzner $H$, Henkes $\mathrm{H}$. Functional outcome and safety of intracranial thrombectomy after emergent extracranial stenting in acute ischemic stroke due to tandem occlusions. Front Neurol 2018;9:940.

6. Sivan-Hoffmann R, Gory B, Armoiry X, Goyal M, Riva R, Labeyrie $P E$, et al. Stent-retriever thrombectomy for acute anterior ischemic stroke with tandem occlusion: a systematic review and meta-analysis. Eur Radio/ 2017;27:247-254.

7. Nogueira RG, Jadhav AP, Haussen DC, Bonafe A, Budzik RF, Bhuva $P$, et al. Thrombectomy 6 to 24 hours after stroke with a mismatch between deficit and infarct. N Engl J Med 2018; 378:11-21.

8. Albers GW, Marks MP, Kemp S, Christensen S, Tsai JP, OrtegaGutierrez $\mathrm{S}$, et al. Thrombectomy for stroke at 6 to 16 hours with selection by perfusion imaging. N Engl J Med 2018;378: 708-718.

9. Higashida RT, Furlan AJ, Roberts H, Tomsick T, Connors B, Barr J, et al. Trial design and reporting standards for intraarterial cerebral thrombolysis for acute ischemic stroke. Stroke 2003;34:e109-e137.

10. Rangel-Castilla L, Rajah GB, Shakir HJ, Shallwani H, Gandhi $\mathrm{S}$, Davies JM, et al. Management of acute ischemic stroke due to tandem occlusion: should endovascular recanalization of the extracranial or intracranial occlusive lesion be done first? Neurosurg Focus 2017;42:E16.

11. Sadeh-Gonik U, Tau N, Friehmann T, Bracard S, Anxionnat R,
Derelle AL, et al. Thrombectomy outcomes for acute stroke patients with anterior circulation tandem lesions: a clinical registry and an update of a systematic review with meta-analysis. Eur J Neurol 2018;25:693-700.

12. Powers WJ, Rabinstein AA, Ackerson T, Adeoye OM, Bambakidis NC, Becker $\mathrm{K}$, et al. Guidelines for the early management of patients with acute ischemic stroke: 2019 update to the 2018 guidelines for the early management of acute ischemic stroke. A guideline for healthcare professionals from the American Heart Association/American Stroke Association. Stroke 2019;50:e344-e418.

13. Demchuk AM, Goyal M, Yeatts SD, Carrozzella J, Foster LD,

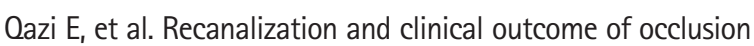
sites at baseline CT angiography in the Interventional Management of Stroke III trial. Radiology 2014;273:202-210.

14. Alawieh A, Kellogg RT, Chatterjee AR, Korson C, Lajthia O, Anadani $M$, et al. Technical and clinical outcomes after thrombectomy for the various segments of the middle cerebral artery. World Neurosurg 2019;128:e445-e453.

Correspondence: Alejandro González García

Interventional Neurorradiology Department, Stroke Research Program, Institute of Biomedicine of Seville (IBiS)/Virgen del Rocio University Hospital, Av Manuel Siurot sn 41013, Seville, Spain

Tel: +34-955012503

Fax: +34-955923101

E-mail: ggjandro@gmail.com

https://orcid.org/0000-0003-0373-7780

Received: July 7, 2020

Revised: October 14, 2020

Accepted: November 16, 2020

This project was partially funded by the ISCIII project PI14/00971. The ITRIBiS project (Improving Translational Research Potential at the Institute of Biomedicine of Seville) has the registration number REGPOT-2013-1. Cooperative Cerebrovascular Disease Research Network (INVICTUS+) (RD16/0019/0015).

The authors have no financial conflicts of interest 
Supplementary Table 1. Baseline characteristics of the whole sample and according to TL vs. no TL presence

\begin{tabular}{|c|c|c|c|c|}
\hline Variable & $\begin{array}{c}\text { All patients } \\
(\mathrm{n}=784,100 \%)\end{array}$ & $\begin{array}{c}\text { TL patients } \\
(n=174,22.2 \%)\end{array}$ & $\begin{array}{l}\text { No TL patients } \\
(n=611,77.8 \%)\end{array}$ & $P$ \\
\hline \multicolumn{5}{|l|}{ General characteristic } \\
\hline Male sex & $410(52.2)$ & $128(73.6)$ & $282(46.2)$ & $<0.001$ \\
\hline Age (yr) & $72(61-80)$ & $63(57-73)$ & 75 (64-82) & $<0.001$ \\
\hline Admission NIHSS & $16(10-21)$ & $16(10-20)$ & $17(11-22)$ & 0.604 \\
\hline Hypertension & $565(72)$ & $126(72.4)$ & 439 (71.8) & 0.884 \\
\hline Diabetes mellitus & $229(29.2)$ & $47(27)$ & $182(29.8)$ & 0.477 \\
\hline Dyslipidemia & $358(45.6)$ & $72(41.4)$ & $286(46.8)$ & 0.205 \\
\hline Smoking habit & 145 (18.5) & 54 (31.3) & $91(14.8)$ & $<0.001$ \\
\hline Atrial fibrillation & $244(31.1)$ & 18 (10.3) & $226(37)$ & $<0.001$ \\
\hline Unknown stroke onset & $249(31.7)$ & $57(32.8)$ & $192(31.4)$ & 0.739 \\
\hline Prior aspirin intake & $238(30.3)$ & $48(27.6)$ & $190(31.1)$ & 0.374 \\
\hline Prior clopidogrel intake & $36(4.6)$ & $10(5.7)$ & $26(4.3)$ & 0.407 \\
\hline Admission ASPECTS & $9(7-10)$ & $8(7-9)$ & $9(7-10)$ & 0.001 \\
\hline Intravenous fibrinolysis & $320(40.8)$ & $87(50)$ & $233(38.1)$ & 0.005 \\
\hline Symptoms onset-intravenous fibrinolysis time ( $\mathrm{min}$ ) & $125(97.5-172.5)$ & $120(95-180)$ & $130(100-170)$ & 0.582 \\
\hline Cardioembolic cause $^{*}$ & $393(50.1)$ & $21(12.3)$ & $372(62.1)$ & $<0.001$ \\
\hline Atheromatous cause* & $179(22.8)$ & $127(74.3)$ & $52(8.7)$ & $<0.001$ \\
\hline DAWN or DEFUSE 3 eligibility patients & $249(31.7)$ & $57(32.8)$ & $192(31.4)$ & 0.739 \\
\hline \multicolumn{5}{|l|}{ Time and procedural characteristic } \\
\hline Door-CT time (min) & $20(15-30)$ & $20(15-35)$ & $20(15-30)$ & 0.483 \\
\hline Door-needle time (min) & $45(35-60)$ & $45(30-60)$ & $50(35-60)$ & 0.280 \\
\hline Door-groin puncture time (min) & $110(75-150)$ & $105(70-160)$ & $110(80-148)$ & 0.625 \\
\hline Groin-recanalization time (min) & $25(13-54)$ & $50(24-80)$ & $21(10-40)$ & $<0.001$ \\
\hline Symptoms onset-groin puncture time (min) & $235(160-375)$ & $245(145-385)$ & $230(160-370)$ & 0.980 \\
\hline Number of passes & $1(1-3)$ & $2(1-3)$ & $1(1-3)$ & 0.588 \\
\hline General anesthesia & $52(6.6)$ & $15(8.7)$ & $37(6.1)$ & 0.473 \\
\hline Carotid stent & 151 (19.2) & $107(61.5)$ & $44(7.2)$ & $<0.001$ \\
\hline Anterior communicating artery & $186(23.7)$ & $104(59.8)$ & $82(13.4)$ & $<0.001$ \\
\hline Posterior communicating artery & $69(8.8)$ & $49(28.2)$ & $20(3.3)$ & $<0.001$ \\
\hline Retrograde ophthalmic artery & $38(4.8)$ & $34(19.5)$ & $4(0.7)$ & $<0.001$ \\
\hline MT clopidogrel & $8(1)$ & $3(1.7)$ & $5(0.9)$ & 0.362 \\
\hline MT reopro ${ }^{+}$ & $38(4.8)$ & $17(9.8)$ & $21(3.4)$ & 0.001 \\
\hline
\end{tabular}

Values are presented as number (\%) or median (interquartile range).

TL, tandem lesion; NIHSS, National Institutes of Heart Stroke Scale; ASPECTS, Alberta Stroke Program Early CT Score; DAWN, DWI or CTP Assessment With Clinical Mismatch in the Triage of Wake-Up and Late Presenting Strokes Undergoing Neurointervention With Trevo; DEFFUSE 3, Endovascular Therapy Following Imaging Evaluation for Ischemic Stroke 3; CT, computed tomography; MT, mechanical thrombectomy.

${ }^{*}$ According to Trial of Org 10172 in Acute Stroke Treatment (TOAST) classification; ${ }^{+}$MT clopidogrel or reopro refers to clopidogrel and reopro administered during mechanical thrombectomy. 
Supplementary Table 2. Baseline characteristics of the sample according to intracranial occlusion

\begin{tabular}{|c|c|c|c|c|c|c|c|}
\hline Variable & $\begin{array}{c}\text { TICA } \\
(n=117,14.9 \%)\end{array}$ & $\begin{array}{c}M 1 \\
(n=278,35.4 \%)\end{array}$ & $\begin{array}{c}M 2 \\
(n=216,27.5 \%)\end{array}$ & $\begin{array}{c}\text { ICA+TICA } \\
(n=36,4.6 \%)\end{array}$ & $\begin{array}{c}\mathrm{ICA}+\mathrm{M} 1 \\
(\mathrm{n}=87,11.1 \%)\end{array}$ & $\begin{array}{c}\text { ICA+M2 } \\
(n=51,6.5 \%)\end{array}$ & $P$ \\
\hline \multicolumn{8}{|l|}{ General characteristic } \\
\hline Male sex & $48(41.4)$ & $120(43.6)$ & $109(50.7)$ & $25(67.6)$ & $62(69.7)$ & 45 (86.5) & $<0.001$ \\
\hline Age (yr) & $74.5(64.5-81)$ & $76(65-82)$ & $73(61-81)$ & $61(48-71)$ & $63(58.5-72)$ & $65(54-76)$ & 0.001 \\
\hline Admission NIHSS & $20(15-23.5)$ & $17(14-21)$ & $11(8-18)$ & $18(17-20)$ & $15(9.5-20.5)$ & $15(9.5-19)$ & $<0.001$ \\
\hline Hypertension & $73(62.9)$ & $202(73.5)$ & $161(74.9)$ & $28(75.7)$ & $66(74.2)$ & $34(65.4)$ & 0.187 \\
\hline Diabetes mellitus & 34 (29.3) & $76(27.6)$ & $71(33)$ & $7(18.9)$ & $24(27)$ & $17(32.7)$ & 0.513 \\
\hline Dyslipidemia & $47(40.5)$ & $130(47.3)$ & $108(50.2)$ & $13(35.1)$ & $38(42.7)$ & $22(42.3)$ & 0.354 \\
\hline Smoking habit & $21(18.1)$ & 41 (14.9) & $27(12.6)$ & $10(27)$ & 30 (33.7) & $16(30.8)$ & $<0.001$ \\
\hline Atrial fibrillation & $49(42.2)$ & 109 (39.6) & $68(31.6)$ & $3(8.1)$ & $7(7.9)$ & $8(15.4)$ & $<0.001$ \\
\hline Unknown stroke onset & $33(28.4)$ & $84(30.5)$ & 72 (33.5) & 15 (40.5) & 21 (23.6) & $24(46.2)$ & 0.073 \\
\hline Prior aspirin intake & 27 (23.3) & $88(32)$ & $74(34.4)$ & $7(18.9)$ & $22(24.7)$ & 19 (36.5) & 0.095 \\
\hline Prior clopidogrel intake & $2(1.7)$ & $13(4.7)$ & $11(5.1)$ & $1(2.7)$ & $3(3.4)$ & $6(11.5)$ & 0.124 \\
\hline Admission ASPECTS & $8(7-9)$ & $9(7-10)$ & $9(8-10)$ & $7(7-8)$ & $8(7-9)$ & $9(8-9)$ & $<0.001$ \\
\hline Intravenous fibrinolysis & $35(30.2)$ & $117(42.5)$ & $80(37.2)$ & $16(43.2)$ & 48 (53.9) & $24(46.2)$ & 0.016 \\
\hline Cardioembolic cause $^{*}$ & $76(65.5)$ & $179(65.8)$ & $117(55.5)$ & $2(5.6)$ & $9(10.6)$ & $10(20)$ & $<0.001$ \\
\hline Atheromatous cause ${ }^{*}$ & $7(6)$ & $30(11)$ & $15(7.1)$ & 29 (80.6) & $64(75.3)$ & $34(68)$ & $<0.001$ \\
\hline \multicolumn{8}{|l|}{ Time and procedural characteristic } \\
\hline Door-CT time (min) & $20(15-30)$ & $20(15-30)$ & $20(15-35)$ & $20(15-20)$ & $20(10-35)$ & $25(20-37.5)$ & 0.033 \\
\hline Door-needle time ( $\mathrm{min}$ ) & $47.5(37.5-82.5)$ & $50(35-60)$ & $50(35-65)$ & $35(25-45)$ & $45(30-62.5)$ & $42(40-67.5)$ & 0.237 \\
\hline Door-groin puncture time (min) & $110(82.5-132.5)$ & $105(75-140)$ & $117.5(85-165)$ & $90(80-190)$ & $100(62.5-152.5)$ & $125(77.5-177.5)$ & 0.667 \\
\hline Groin-recanalization time (min) & $42(23-70)$ & $15(10-35)$ & $23(11-41)$ & $50(25-80)$ & $35(20-72.5)$ & $50(30-89.5)$ & $<0.001$ \\
\hline Number of passes & $3(2-5.5)$ & $1(1-2)$ & $1.5(1-2)$ & $2(2-3)$ & $2(1-3)$ & $1(1-3.5)$ & $<0.001$ \\
\hline General anesthesia & $10(8.6)$ & $17(6.2)$ & $8(3.7)$ & $3(8.1)$ & $8(9.1)$ & $5(9.6)$ & 0.674 \\
\hline Carotid stent & $0(0)$ & $0(0)$ & $0(0)$ & $23(62.1)$ & $49(55)$ & $30(57.7)$ & $<0.001$ \\
\hline Anterior communicating artery & $55(47.4)$ & $19(6.9)$ & $3(1.4)$ & $23(62.2)$ & $46(51.7)$ & $39(75)$ & $<0.001$ \\
\hline Posterior communicating artery & $9(7.8)$ & $6(2.2)$ & $2(0.9)$ & $7(18.9)$ & $24(27)$ & $20(38.5)$ & $<0.001$ \\
\hline MT clopidogrel ${ }^{+}$ & $1(0.9)$ & $2(0.7)$ & $2(0.9)$ & $1(2.7)$ & $1(1.1)$ & $1(1.9)$ & 0.876 \\
\hline MT reopro ${ }^{+}$ & $3(2.6)$ & $12(4.4)$ & $4(1.9)$ & $2(5.4)$ & $10(11.2)$ & $6(11.5)$ & 0.002 \\
\hline
\end{tabular}

Values are presented as number (\%) or median (interquartile range).

TICA, terminal internal carotid artery; ICA, internal carotid artery; NIHSS, National Institutes of Heart Stroke Scale; ASPECTS, Alberta Stroke Program Early CT Score; CT, computed tomography; MT, mechanical thrombectomy.

${ }^{*}$ According to Trial of Org 10172 in Acute Stroke Treatment (TOAST) classification; ${ }^{+}$MT clopidogrel or reopro refers to clopidogrel and reopro administered during MT. 
Supplementary Table 3. Safety and efficacy results in no TL vs. TL after stroke (atherothrombotic vs. cardioembolic) subtype adjustment

\begin{tabular}{|c|c|c|c|c|c|c|}
\hline \multirow{2}{*}{ Variable } & \multicolumn{3}{|c|}{ Atherothrombotic } & \multicolumn{3}{|c|}{ Cardioembolic } \\
\hline & No TL & $\mathrm{TL}$ & $P$ & No TL & $\mathrm{TL}$ & $P$ \\
\hline $\mathrm{TICl} 2 \mathrm{~b}-3$ & $43(82.7)$ & 117 (93.6) & 0.025 & $352(94.6)$ & $18(85.7)$ & 0.091 \\
\hline mRS 0-2 & 19 (42.2) & 67 (57.8) & 0.137 & $171(56.6)$ & $6(42.9)$ & 0.414 \\
\hline Death & $3(5.8)$ & 15 (11.8) & 0.222 & $31(8.3)$ & $4(19)$ & 0.094 \\
\hline sICH & $4(7.7)$ & $6(4.7)$ & 0.432 & 11 (3) & $2(9.5)$ & 0.102 \\
\hline
\end{tabular}

Values are presented as number (\%).

$\mathrm{TL}$, tandem lesion; $\mathrm{TICl}$, thrombolysis in cerebral ischemia; mRS, modified Rankin Scale; sICH, symptomatic intracranial hemorrhage.

Supplementary Table 4. Intracranial hemodynamic compensations rates (\%) according to site occlusion

\begin{tabular}{|c|c|c|c|c|c|c|}
\hline Variable & TICA vs. ICA+TICA & $P$ & $\mathrm{M} 1$ vs. $\mathrm{ICA}+\mathrm{M} 1$ & $P$ & $\mathrm{M} 2$ vs. ICA+M2 & $P$ \\
\hline Retrograde ophthalmic artery & 4.2 vs. 19 & 0.044 & 0.9 vs. 25.8 & $<0.001$ & 0 vs. 31.7 & $<0.001$ \\
\hline Anterior communicating artery & 62.9 vs. 78.6 & 0.125 & 19.3 vs. 62.9 & $<0.001$ & 6.5 vs. 86.4 & $<0.001$ \\
\hline Posterior communicating artery & 13 vs. 33.3 & 0.038 & 6.1 vs. 36.4 & $<0.001$ & 4.7 vs. 51.4 & $<0.001$ \\
\hline Collateral flow grade $0-2$ & 58 vs. 64.7 & 0.488 & 46 vs. 48.1 & 0.735 & 56.5 vs. 47.9 & 0.278 \\
\hline Collateral flow grade $3-4$ & 42 vs. 35.3 & 0.488 & 54 vs. 51.9 & 0.735 & 43.5 vs. 52.1 & 0.278 \\
\hline
\end{tabular}

The angiographic collateral grade was evaluated according to the American Society of Interventional and Therapeutic Neuroradiology/Society of Interventional Radiology Collateral Flow Grading System on baseline angiography.

TICA, terminal internal carotid artery; ICA, internal carotid artery. 